\title{
Hemodynamic effects of continuous intravenous injection and bolus plus continuous intravenous injection of oxytocin in cesarean section
}

\author{
Tae-Sung Kim, Jun-Seok Bae, Jung-Man Park, and Sin-Kyu Kang \\ Department of Anesthesiology and Pain Medicine, St. Mary's Hospital, Busan, Korea
}

Background: Oxytocin may cause adverse cardiovascular effects, including tachycardia and hypotension, whereas the optimal dose of oxytocin at elective cesarean section is unclear. To determine the lowest effective dose of oxytocin, we studied the hemodynamic effects of three doses during spinal anesthesia for elective single cesarean delivery.

Methods: Sixty women received oxytocin by continuous $(0.5 \mathrm{IU} / \mathrm{min})$ or bolus-continuous ( 2 or $5 \mathrm{IU}$ prior to 0.25 $\mathrm{IU} /$ min continuous intravenous injection) intravenous injection after clamping of the umbilical cord. We compared changes in heart rate (HR), mean arterial pressure (MAP) and estimated blood loss (EBL). Uterine tone (UT) was assessed by palpation on a linear analog scale (LAS) at 5, 10, 15, 20 and 25 minutes after the oxytocin injection. In addition, oxytocin-related side-effects such as nausea and vomiting were recorded.

Results: Marked hemodynamic changes such as HR and MAP occurred in the bolus-continuous groups but not in the continuous groups. Although we were not able to observe a variation of EBL in each group, the UT significantly increased in the bolus-continuous groups when compared with that the continuous groups. In addition, the hemodynamic changes such as HR and MAP were lower in the two IU bolus-continuous group than those in the five IU group.

Conclusions: Although bolus-continuous injection of oxytocin resulted in more hemodynamic changes than continuous injection, bolus-continuous injection had a greater effect on uterine contraction. Furthermore, two IU bolus-continuous injection showed lower hemodynamic changes than in the five IU bolus-continuous injection. (Korean J Anesthesiol 2011; 61: 482-487)

Key Words: Bolus-continuous intravenous injection, Continuous intravenous injection, Elective cesarean section, Oxytocin, Spinal anesthesia.

Received: March 22, 2011. Revised: 1st, May 16, 2011; 2nd, June 3, 2011. Accepted: June 7, 2011.

Corresponding author: Jun-Seok Bae, M.D., Department of Anesthesiology and Pain Medicine, St. Mary' s Hospital, 538-41, Yongho-dong, Nam-gu, Busan 608-838, Korea. Tel: 82-51-933-7363, Fax: 82-51-932-8600, E-mail: soaf29@naver.com

(c) This is an open-access article distributed under the terms of the Creative Commons Attribution Non-Commercial License (http:// creativecommons.org/licenses/by-nc/3.0/), which permits unrestricted non-commercial use, distribution, and reproduction in any medium, provided the original work is properly cited. 


\section{Introduction}

The hemodynamic changes in parturient women after cesarean delivery may be caused by the elimination of the aorta-caval compression, autotransfusion of uterine contraction, hemorrhage, and vasoconstriction and excitation, but some studies have reported that uterotonic drug is the main factor [1,2]. Uterotonic drug, which is most frequently used for cesarean section, is oxytocin, which induces uterine contraction during cesarean section and peripheral vasodilation along with a decrease in arterial pressure after delivery and thus, reduces hemorrhage $[3,4]$. However, intravenous injection of oxytocin during cesarean section may cause cardiovascular side effects such as tachycardia or hypotension $[1,2]$ and is reported to cause even cardiovascular collapse and death in the most severe cases $[5,6]$. Moreover, various other side effects have been reported including fluid pooling or pulmonary edema by the antidiuretic effect of oxytocin [7-9].

Hence, determining the oxytocin concentration for cesarean section is important. However, since the activity of oxytocin varies from individual to individual [10] and an appropriate dose has not been established, 5-20 IU is intravenously injected based on experience $[11,12]$. There is a recent assertion that bolus intravenous injection or bolus-continuous parallel intravenous injection of oxytocin is more effective than continuous intravenous injection in cesarean section $[13,14]$. There is also a report that a great quantity of bolus intravenous injection requires caution because it causes hemodynamic changes related to hypotension $[1,2]$. Therefore, research is necessary on the method of oxytocin injection and the effective minimum concentration for cesarean section to induce uterine contraction without side effects.

In this study, we did continuous intravenous injection or bolus-continuous intravenous injection of oxytocin after clamping of the umbilical cord during planned cesarean sections under spinal anesthesia, and investigated the uterine tone (TU) and hemodynamic changes.

\section{Materials and Methods}

This study was approved by the Hospital Ethics Committee. The subjects of this study were 60 singleton gestation pregnant women who were 38 weeks or more into their pregnancy, and were classified as Class 1 or 2 according to the American Society of Anesthesiologist (ASA) physical status classification and were supposed to undergo planned cesarean section under spinal anesthesia in the obstetrics and gynecology department in our hospital. The subjects were visited before the operation and written consent was given by the subjects. Pregnant women who had contraindication for spinal anesthesia, those whose weight was $100 \mathrm{~kg}$ or higher, those who had fetal abnormalities, diabetes, gestational hypertension, cardiovascular disease, or cerebral hemorrhage, and those whose labor pain had already started were excluded from the study. The age, height, and weight of the patients were recorded.

About $500 \mathrm{ml}$ of Hartmann solution was rapidly dripped into all the parturient women before inducing anesthesia. An electrocardiograph, automated noninvasive blood pressure device, and a pulse oximeter were attached to the subjects, and oxygen was injected at $5 \mathrm{~L} / \mathrm{min}$ through a mask. For the spinal anesthesia, the L3-4 or L4-5 lumbar region was punctured with a $25 \mathrm{G}$ Quincke needle when the parturient women were in the left lateral recumbent position, and $0.5 \%$ hyperbaric bupivacaine solution (10-12 $\mathrm{mg}$ ) was injected after verifying the cerebrospinal fluid leakage. Immediately after the anesthesia, the parturient women were asked to take the supine position and the maximum sensory block level was set to T4-6. The blood pressure and HR were measured in five-minute intervals from the time just after the anesthesia started to the end of the operation. However, during the 10 minutes after the oxytocin was injected, the blood pressure and HR were measured in one minute intervals, and delivery of the newborn was set as the baseline. The parturient women were asked if they felt or experienced nausea and vomiting after oxytocin injection and the results were recorded. When some of the parturient women had severe vomiting or continual nausea, the antiemetic drug, ondansetron $8 \mathrm{mg}$, was intravenously injected. In the cases where the systolic pressure decreased by more than $20 \%$ compared to the blood pressure before coming to the operating room, $10 \mathrm{mg}$ of ephedrine was intravenously injected until the blood pressure was normalized, and these cases were excluded from the study.

The subjects were randomly divided into three groups, 20 subjects per group. For the oxytocin injection, $5 \mathrm{ml}$ of bolus diluted with normal saline and $40 \mathrm{ml}$ of infusion solution were prepared in advance by a nurse who did not participate in the study. In Group 1, bolus intravenous injection was done for about five seconds with $5 \mathrm{ml}$ of normal saline and then continuous intravenous injection of $20 \mathrm{IU}$ oxytocin diluted with $40 \mathrm{ml}$ of normal saline was done at a rate of $0.5 \mathrm{IU} / \mathrm{min}$ for 40 minutes with a syringe pump. In groups 2 and 3, bolus intravenous injection of 2 IU and 5 IU of oxytocin diluted with $5 \mathrm{ml}$ of normal saline was done at a rate of $1 \mathrm{ml} / \mathrm{sec}$, and then continuous intravenous injection of $10 \mathrm{IU}$ of oxytocin diluted with $40 \mathrm{ml}$ of normal saline was done at a rate of $0.25 \mathrm{IU} /$ min for 40 minutes with a syringe pump. The epidemiological data did not show a difference in the age, height, weight, and gestation period of the patients among the groups (Table 1). The obstetrician evaluated the degree of uterine contraction on a LAS in which the grade was determined from a grade of o to 10 
Table 1. Maternal Demographic and Anesthetic Data

\begin{tabular}{lrrr}
\hline & Group 1 & Group 2 & Group 3 \\
\hline Age (yr) & $30.5 \pm 3.5$ & $31.1 \pm 3.1$ & $31.0 \pm 2.8$ \\
Weight (kg) & $67.5 \pm 6.2$ & $69.2 \pm 6.7$ & $68.8 \pm 5.9$ \\
Height (cm) & $159.2 \pm 4.0$ & $159.5 \pm 3.8$ & $159.7 \pm 4.2$ \\
Gestational age (weeks) & $39.0 \pm 0.7$ & $38.7 \pm 0.7$ & $38.6 \pm 1.0$ \\
\hline
\end{tabular}

$\mathrm{N}=20$ in each group. Values are mean \pm SD. Oxytocin was injected in the following doses; Group 1: $0.5 \mathrm{IU} / \mathrm{min}$ continuous injection, Group 2: 2 IU bolus-continuous injection, Group 3: 5 IU boluscontinuous injection. There was no significant difference among threegroups.

( 0 = completely atonic, $10=$ fully contracted ) [15] at five-minute intervals during the operation for 25 minutes from the time just after the newborn was delivered. In the cases where the uterine contraction was considered as insufficient during the operation, intravenously injection of $0.2 \mathrm{mg}$ of methylergonovine was done by the request of the obstetrician, diluting it with normal saline and slowly injecting it over one to two minutes with careful monitoring of the blood pressure. The blood loss was estimated by measuring the weight of the suction bottle and gauze excluding the amniotic fluid.

All the measured values obtained in the experiment were presented as the mean \pm standard deviation and 'n.' ANOVA was done to compare age, weight, height, gestational age, fluid injection, and blood loss among the groups. An ANOVA was also performed to compare the HR and MAP among the group. To compare the degree of uterine contraction among the groups, Kruskal-Wallis test, a nonparametric method, was done. A repeated measures ANOVA was done to compare the HR, MAP, and degree of uterine contraction within a group. The Bonferroni method was used for the post-hoc test of the $\mathrm{HR}$ and MAP, and the Bonferroni correction was done for the post-hoc test of the degree of uterine contraction. A chi-square correlation analysis was done for each group to compare the frequency of vomiting and nausea and the number of times an antiemetic drug was used. All the statistical analyses were done with SPSS 15.0 (SPSS Inc., Chicago, IL, USA), with a significance level of 0.05 .

\section{Results}

There was no significant difference in the age, weight, height, and gestational age among the groups $(\mathrm{P}>0.05)$. Hemorrhage was $457.1 \pm 15.5 \mathrm{ml}$ in Group 1, $450.5 \pm 13.9 \mathrm{ml}$ in Group 2, and $446.2 \pm 13.6 \mathrm{ml}$ in Group 3, with no significant difference (P > 0.05 , Table 1$)$. The quantity of fluid injected during the operation was almost the same in all the groups, with no significant difference. The number of parturient women who experienced nausea and vomiting after intravenous injection of oxytocin
Table 2. Anesthetic Maternal Demographic Data

\begin{tabular}{lccc}
\hline & Group 1 & Group 2 & Group 3 \\
\hline Intravenous fluid (ml) & $1,897.3 \pm 107.9$ & $1,962.0 \pm 133.8$ & $1,886.8 \pm 83.0$ \\
EBL (ml) & $457.1 \pm 15.5$ & $450.5 \pm 13.9$ & $446.2 \pm 13.6$ \\
Additional uterotonic & 6 & 2 & 1 \\
$\quad$ treatment (n) & 2 & 3 & 5 \\
Nausea (n) & 1 & 1 & 3 \\
Vomiting (n) & 1 & 1 & 3 \\
Antiemetic treatment (n) & & & \\
\hline
\end{tabular}

$\mathrm{N}=20$ in each group. Values are mean \pm SD and No. of patients for the others. Oxytocin was injected in the following doses; Group 1: $0.5 \mathrm{IU} / \mathrm{min}$ continuous injection, Group 2: 2 IU bolus-continuous injection, Group 3: 5 IU bolus-continuous injection. There was no significant difference among three groups.

Table 3. Hemodynamic Change Data

\begin{tabular}{cccc}
\hline & Group 1 & Group 2 & Group 3 \\
\hline MAP (mmHg) & & & \\
Before oxytocin & 93 & 93 & 94 \\
At max. change & 89 & 86 & $83^{*}$ \\
HR (beats/min) & & & \\
Before oxytocin & 93 & 92 & 93 \\
At max. change & 98 & 107 & $118^{*}$ \\
\hline
\end{tabular}

$\mathrm{N}=20$ in each group. Values are maternal mean arterial pressure (MAP) and heart rate (HR). Measurements were performed before oxytocin and at the time of maximun changes. Oxytocin was injected in the following doses; Group 1: $0.5 \mathrm{IU} / \mathrm{min}$ continuous injection, Group 2: 2 IU bolus-continuousinjection, Group 3: 5 IU bolus-continuous injection. ${ }^{*} \mathrm{P}<0.05$ compared to before oxytocin injection.

and had antiemetic drugs administered was greater in groups 1 and 2 than in Group 3, but there was no significant difference among the groups $(\mathrm{P}>0.05)$. On the other hand, additional intravenous injection of uterotonic drug was required more in groups 2 and 3 than in Group1, but there was no significant difference among the groups ( $\mathrm{P}>0.05$, Table 2$)$.

The MAP was similar before the oxytocin intravenous injection. A maximum decrease of $4.5 \%$ was observed in Group 1 two minutes after intravenous injection of oxytocin whereas it was $7.6 \%$ and $11.8 \%$ in groups 2 and 3, respectively, one minute after injection. However, the comparison within the groups showed that the maximum decrease was significant only in Group 3 (P < 0.05 , Table 3 ). There was no case where a hypertensor was used because of hypotension. The MAP decreased after intravenous injection of oxytocin and recovered after four minutes in each group. The comparison among the groups showed that the decrease in groups 2 and 3 at one minute after the injection was significantly greater than that in Group 1 , and the decrease in Group 3 was significantly greater than that in Group $2(\mathrm{P}<0.05$, Fig. 1).

The HR in each group was similar before the intravenous injection of oxytocin. A maximum increase of $7.6 \%$ was observed 


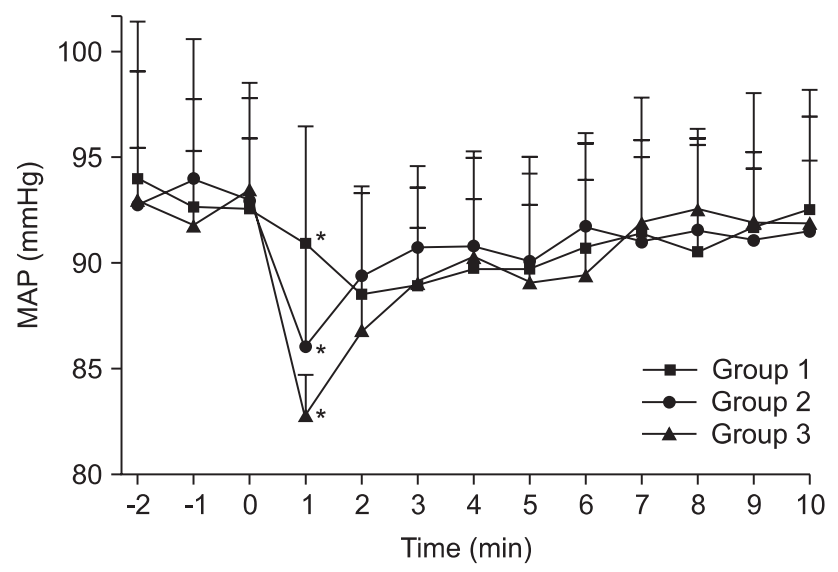

Fig. 1. Change of maternal mean arterial pressure (MAP) after oxytocin injection during Cesarean delivery. Oxytocin was injected in the following doses; Group 1: $0.5 \mathrm{IU} / \mathrm{min}$ continuous injection, Group 2: 2 IU bolus-continuous injection, Group 3: 5 IU boluscontinuous injection. ${ }^{*} \mathrm{P}<0.05$ compared with each group after oxytocin injection.

in Group 1 two minutes after intravenous injection of oxytocin while it was $17.0 \%$ and $26.1 \%$ in groups 2 and 3, respectively, one minute after the injection, but the increase was significant only in Group 3 ( $\mathrm{P}<0.05$, Table 3 ). The comparison among the groups showed that the increase at one, two and three minutes after the injection was significantly greater in groups 2 and 3 than that in Group 1, and the increase in Group 3 was significantly greater than that in Group 2. The increased HR recovered after five minutes in each group.

The degree of uterine contraction at five and ten minutes after the injection of oxytocin was significantly higher in groups 2 and 3 than that in Group 1 according to the LAS score based on the palpation of the obstetrician $(\mathrm{P}<0.05)$. Particularly in Group 1, the LAS score at five minutes after the injection of oxytocin was 8.0, indicating the failure to obtain an appropriate degree of uterine contraction, and thus, another $30 \%$ of additional uterotonic drug by intravenous injection was required. All the groups maintained an appropriate state of uterine contraction at ten minutes after the injection, and thereafter, no uterotonic drug by intravenous injection was additionally done because of re-relaxation.

\section{Discussion}

Oxytocin, a hormone that is secreted from the pituitary posterior lobe, decreases blood pressure by causing peripheral vasodilation and increasing the $\mathrm{HR}$ and induces uterine contraction $[3,4]$. Uterine contraction by oxytocin is enhanced since there are more intrauterine oxytocin receptors [16]. The oxytocin sensitivity of pregnant women reaches the maximum in the full term of the pregnancy because of the increase of intrauterine oxytocin receptors by gestational estrogen [10]. Hence, during normal delivery, the oxytocin discharged from the pituitary posterior lobe by the obstetric canal stimulus of the fetus can induce labor pain and sufficient uterine contraction with an extreme low-dose of $10 \mathrm{mIU} / \mathrm{min}$ [17]. In the case of a planned cesarean section, however, a relatively high-dose of oxytocin (5-20 IU) is intravenously injected since the action of the oxytocin is not normal $[11,12]$. Since hemodynamic changes are proportional to the intravenously injected dose of oxytocin, studies have been done on effective concentrations and intravenous injection methods.

Sarna et al. [11] reported that oxytocin infusion at a rate of $1 \mathrm{IU} / \mathrm{min}$ did not show any difference in uterine contraction and blood loss with that of 5 IU by intravenous injection even though the total dose may have been more than 5 IU. Kim et al. [12] published the same assertion. On the contrary, Zarzur [18] recommended an infusion at a rate lower than $1 \mathrm{IU} / \mathrm{min}$ because infusion at a rate higher than $0.25 \mathrm{IU} / \mathrm{min}$ may cause such symptoms as hemangiectatic hypotension, tachycardia, increased cardiac output, and myocardial ischemia. In Group 1 , in this study, where the infusion was done at the rate of 0.5 $\mathrm{IU} / \mathrm{min}$, there was no significant hemodynamic change due to the infusion of oxytocin, but about another $30 \%$ of additional uterotonic drug was required for parturient women to obtain an appropriate degree of uterine contraction.

According to many recent studies, oxytocin bolus injection was reported to be more effective than infusion by intravenous injection to reduce blood loss by inducing the appropriate uterine contraction [13], and thus, the minimum bolus dose has been discussed [19-21]. A suggested method to reduce hemodynamic changes is the repeated intravenous injections of a small quantity of a bolus dose and bolus-continuous parallel intravenous injection [14,22]. Svanström et al. [6] stated that attention is required because a bolus injection of $10 \mathrm{IU}$ oxytocin may cause temporary hypotension and tachycardia as well as myocardial ischemia, apart from the operation, pregnancy, and autonomic blocking by spinal anesthesia. Pinder et al. [2] recommended $5 \mathrm{IU}$ as the bolus dose since $10 \mathrm{IU}$ of bolus intravenous injection caused severe hemodynamic changes, but they stated that the dose might not be considered as safe, either. On the contrary, Butwick et al. [20] asserted that a dose of more than $5 \mathrm{IU}$ might not be necessary because appropriate uterine contraction occurred even with a bolus dose of $0.5-$ 3 IU. Sartain et al. [14] injected 2 IU and 5 IU of bolus doses, which are the same doses for groups 2 and 3 in our experiment and reported that the use of 2 IU was more effective because the hemodynamic changes were more severe in the 5 IU group even though the uterine contraction were similar in the two groups. These results were similar to our results and the hemodynamic changes were always temporary. 


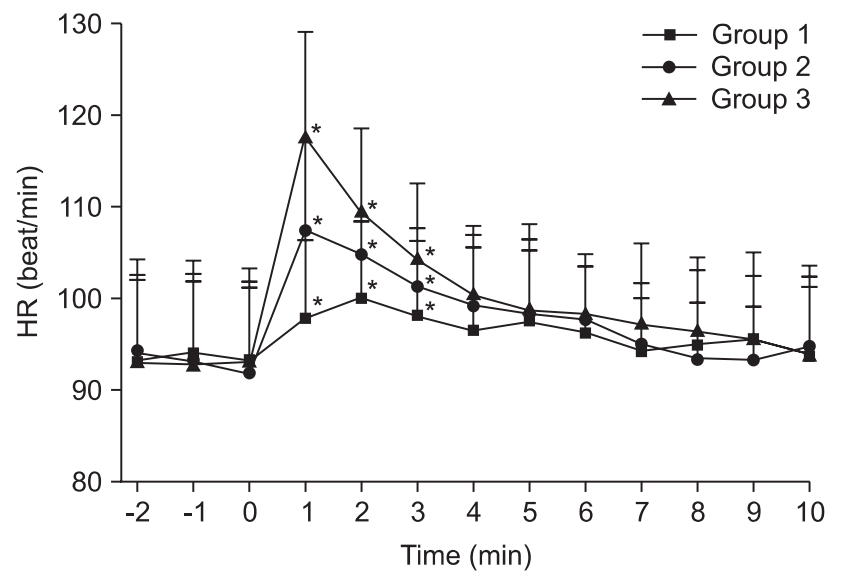

Fig. 2. Change of heart rate (HR) after oxytocin injection during Cesarean delivery. Oxytocin was injected in the following doses; Group 1: $0.5 \mathrm{IU} / \mathrm{min}$ continuous injection, Group 2: 2 IU boluscontinuous injection, Group 3: 5 IU bolus-continuous injection. ${ }^{*} \mathrm{P}<$ 0.05 compared with each group after oxytocin injection.

In the case of Group 3 in which 5 IU bolus-continuous intravenous injection was done, the MAP and HR were significantly decreased by $11.8 \%$ and significantly increased by $26.1 \%$, respectively, at one minute after the injection of oxytocin. On the contrary, the MAP and HR were not significantly changed in Group 2 in which 2 IU bolus-continuous intravenous injection was done (Fig. 1, 2, Table 3). Thus, it was found that the hemodynamic changes varied by the bolus injected oxytocin dose and changes were recovered from in about 4-5 minutes after oxytocin injection. As shown in this study, delivery of the newborn and the following excitement may affect the HR because parturient women are conscious during cesarean section under spinal anesthesia. However, the main factor of the changes may be the effect of oxytocin itself.

The half-life of oxytocin is about 5-12 minutes and a blood steady state is reached in about $30-60$ minutes after intravenous injection $[23,24]$. Thus, the oxytocin dose and injection method play an important role in the hemodynamic changes before a steady state is reached. These hemodynamic changes by oxytocin are always temporary and the effect on healthy parturient women is small. However, they may be dangerous to parturient women who have hypovolemia or cardiovascular diseases. Hence, continuous intravenous injection of oxytocin at a low concentration is primarily recommended as a safe injection method for parturient women in a high-risk group $[1,2]$. Nevertheless, repeated bolus intravenous injection with a small quantity of bolus or parallel intravenous injection of a small quantity of bolus plus continuous injection is also used because of the rapid desensitization by which the hemodynamic changes are significantly decreased in the repeated oxytocin than in the initial injection $[14,22]$. In our study, a longer time

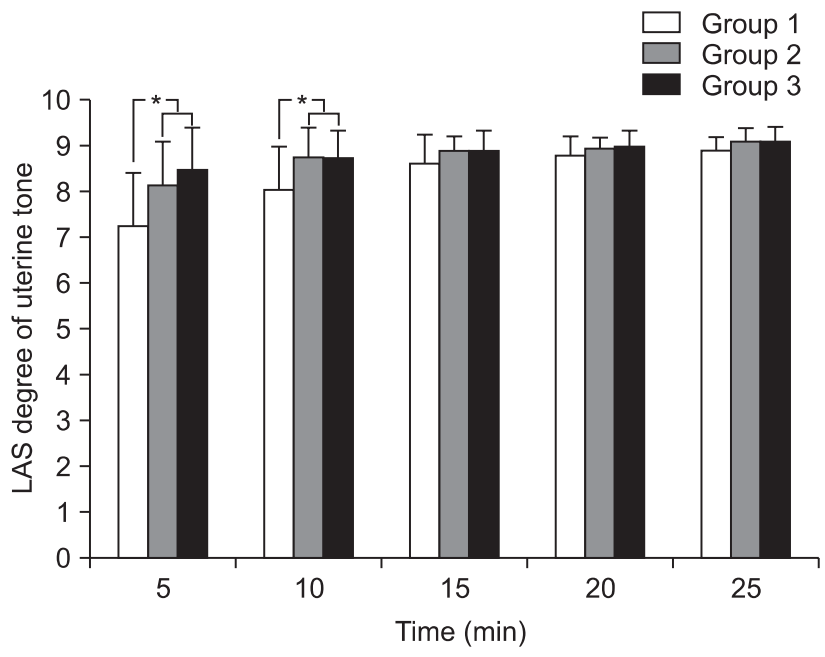

Fig. 3. Prevalence of adequate uterine tone (UT) by linear analog scale (LAS) after oxytocin injection during Cesarean delivery. Oxytocin was injected in the following doses; Group 1: $0.5 \mathrm{IU} / \mathrm{min}$ continuous injection, Group 2: 2 IU bolus-continuous injection, Group 3: 5 IU bolus-continuous injection. Numerical scores for UT were assessed by the obstetrician using a linear analog scale of 0 (absent UT) to 10 (optimal UT). *P $<0.05$ compared with each group after oxytocin injection.

was taken in the continuous intravenous injection group (Group 1) to induce the appropriate uterine contraction with a LAS score of 8.0 than the bolus-continuous parallel intravenous injection groups (groups 2 and 3), and about another $30 \%$ of additional uterotonic drug was required in Group 1, but there was no significant difference in the mean blood loss. Nausea and vomiting occurred more in Group 3, but the difference was not significant (Table 2, Fig. 3).

In conclusion, with parturient women undergoing planned cesarean section under spinal anesthesia, infusion of $20 \mathrm{IU}$ of oxytocin was done at a rate of $0.5 \mathrm{IU} / \mathrm{min}$, and $2 \mathrm{IU}$ or $5 \mathrm{IU}$ bolus intravenous injection followed by 10 IU oxytocin intravenous injection at a rate of $0.25 \mathrm{IU} / \mathrm{min}$ were done immediately after clamping of the umbilical cord following delivery of the new born, and the results show that a more effective uterine contraction occurred in the bolus-continuous parallel intravenous injection group accompanied by a decreased MAP and increased mean HR which were significantly higher in the 2 IU bolus-continuous parallel intravenous injection group than in the 5 IU bolus-continuous parallel intravenous injection group. These changes may have little effect on healthy parturient women because they can recover within about 4-5 minutes after intravenous injection of oxytocin, as in this study, but the methods that cause the changes are difficult to interpret in women who have uterine atony such as premature separation of placenta or placenta accrete. Although a low-dose bolus intravenous injection may be appropriate to meet the demand 
of obstetricians who want rapid uterine contraction, they should be careful when using this method in parturient women who are suspected to have hypovolemia or cardiovascular diseases because bolus-continuous parallel intravenous injection of 2 IU oxytocin is accompanied by hemodynamic changes in MAP and HR though they may be relatively mild. Further studies need to be conducted on low-dose intravenous injection with less than 2 IU oxytocin in order to obtain more stable and objective data.

\section{References}

1. Thomas JS, Koh SH, Cooper GM. Haemodymainc effects of oxytocin given as i.v. bolus or infusion on women undergoing Caesarean section. Br J Anaesth 2007; 98: 116-9.

2. Pinder AJ, Dresner M, Calow C, Shorten GD, O’Riordan J, Johnson R. Haemodymanic changes caused by oxytocin during caesarean section under spinal anaesthesia. Int J Obstet Anesth 2002; 11: 156-9.

3. Shyken JM, Petrie RH. The use of oxytocin. Clin Perinatol 1995; 22: 907-31.

4. Petersson M. Cardiovascular effects of oxytocin. Prog Brain Res 2002; 139: 281-8.

5. Cooper GM, Lewis G, Neilson J. Confidential enquiries into maternal deaths, 1997-1999. Br J Anaesth 2002; 89: 369-72.

6. Svanström MC, Biber B, Hanes M, Johansson G, Näslund U, Bålfors EM. Signs of myocardial ischemia after injection of oxytocin: a randomized double-blind comparison of oxytocin and methylergometrine during Caesarean section. Br J Anaesth 2008; 100: 683-9.

7. Edwards BR, LaRochelle FT Jr. Antidiuretic effect of endogenous oxytocin in dehydrated Brattleboro homozygous rats. Am J Physiol 1984; 247: F453-65.

8. Heytens L, Camu F. Pulmonary edema during cesarean section related to the use of oxytocic drugs. Acta Anaesthesiol Belg 1984; 35 : 155-64.

9. Shahin J, Guharoy SR. Pulmonary edema possibly developing secondary to the intravenous administration of oxytocin. Vet Hum Toxicol 1991; 33: 587-8.

10. Kimura T, Tanizawa O, Mori K, Brownstein MJ, Okayama H. Structure and expression of a human oxytocin receptor. Nature 1992; 356: 526-9.
11. Sarna MC, Soni AK, Gomez M, Oriol NE. Intravenous oxytocin in patients undergoing elective cesarean section. Anesth Analg 1997; 84: 753-6.

12. Kim DY, Han JI, Chung RY, Lee CH. The effect of three different dosed of intravenous oxytocin on patients undergoing elective Cesarean section. Korean J Anesthesiol 2000; 38: 476-80.

13. Davies GA, Tessier JL, Woodman MC, Lipson A, Hahn PH. Maternal hemodynamics after oxytocin bolus compared with infusion in the third stage of labor: a randomized controlled trial. Obstet Gynecol 2005; 105: 294-9.

14. Sartain JB, Barry JJ, Howat PW, McCormack DI, Bryant M. Intravenous oxytocin bolus of 2 units is superior to 5 units during elective Caesarean section. Br J Anaesth 2008; 101: 822-6.

15. Ghaly RG, Flynn RJ, Moore J. Isoflurane as an alternative to halothane for caesarean section. Anaesthesia 1988; 43: 5-7.

16. Wray S. Uterine contraction and physiological mechanisms of modulation. Am J Physiol 1993; 264: C1-18.

17. Xenakis EM, Langer O, Piper JM, Conway D, Berkus MD. Low-dose versus high-dose oxytocin augmentation of labor - a randomized trial. Am J Obstet Gynecol 1995; 173:1874-8.

18. Zarzur E. Intravenous oxytocin in patients undergoing elective cesarean section. Anesth Analg 1998; 86: 1334.

19. Balki M, Ronayne M, Davies S, Fallah S, Kingdom J, Windrim R, et al. Minimum oxytocin dose requirement after cesarean delivery for labor arrest. Obstet Gynecol 2006; 107: 45-50.

20. Butwick AJ, Coleman L, Cohen SE, Riley ET, Carvalho B. Minimum effective bolus dose of oxytocin during elective Caesarean delivery. Br J Anaesth 2010; 104: 338-43.

21. Carvalho JC, Balki M, Kingdom J, Windrim R. Oxytocin requirements at elective cesarean delivery: a dose-finding study. Obstet Gynecol 2004; 104: 1005-10.

22. Langesaeter E, Rosseland LA, Stubhaug A. Haemodynamic effects of repeated doses of oxytocin during Caesarean delivery in healthy parturients. Br J Anaesth 2009; 103: 260-2.

23. Rall TW. Oxytocin, prostaglandins, ergot alkaloids and other drugs; tocolytic agents. In: Gillman AG, Rall TW, Nies AS, Taylor I', eds. The pharmacological basis of therapeutics. New York, Pergamon Press Inc. 1990, pp 933-53.

24. Crall HD, Mattison DR. Oxytocin pharmacodymanics: effect of long infusions on uterine activity. Gynecol Obstet Invest 1991; 31: 17-22. 